\title{
Introduction: \\ The Empirical and the Formal-Tensions in Scientific Knowledge
}

In the early modern period when many researchers were proposing new approaches for investigating nature, they put two different goals in the forefront: On the one hand, they argued for broad empirical inquiries supported by massive data collections, while on the other they highlighted the ideal of a thorough mathematical treatment and establishing of formal structures. How these two strands were related, if at all, was not so clear-what they shared was essentially the claim to overcome former, purportedly barren traditions and to create new approaches. Indeed, even a brief look into the early years of the Philosophical Transactions suggests that broad empirical data gathering (claimed to be Baconian) and mathematical structure (as in the studies of the laws of collision, for example) had little to do with each other.

These two characteristics of modern science have been with us ever since. While the focus on empirical research justifies our expectation that the basic statements of science be realistic, the mathematical structure aims at guaranteeing them a high degree of rigor and reliability. When Thomas Kuhn introduced his famous dichotomy between 'mathematical' and 'Baconian' sciences, he drew attention to a distinction that had been visible for many centuries. Although his dichotomy has to be 'taken with a pinch of salt', historians of science tend to agree that scientific developments differ widely when it comes to the relationship between the empirical and the formal. There are scientific domains in which the empirical side was always prevailing. Natural history, for example, represents a type of scientific knowledge that consists of huge bodies of detailed information about specific things in all their variations, typically with the claim of being comprehensive for the empirical domain in question. In other cases, such as chemistry, such knowledge is even formulated in quantitative laws. And in general the empirical approach is highly apt for practical use. Recipes describing how to handle specific things can often be easily extracted from this kind of knowledge. It is not by chance, therefore, that major parts of the knowledge of engineering traditions are of an empirical nature.

At the same time, there have also been branches of research in which highly formalized, but less empirical knowledge formed the core, i.e. in which mathematical structures have 
dominated at the cost of the empirical details, and empirical results would only be used and searched selectively. Traditional mathematical domains, such as musical harmony or traditional optics as well as the 'rational mechanics' of the 18th century provide illustrative cases. More recently the development of string theory, and also general evolutionary theory, could be considered domains that have developed a formalism, while their relation to empirical research remains rather open.

However, such a dichotomic understanding of modern science and its history has its limitations. There have always been domains and episodes that do not fit into the Kuhn-type distinction, but in which the empirical and the formal were tightly interwoven in different ways. It is research of this nature that is the focus of this special issue. In the early modern period (and, in fact, throughout its early history), astronomy provides a striking case of how the empirical may become integrated with the formal. Kepler, for example, used a large bulk of empirical data to fundamentally reform a highly developed mathematical domain. And for any modern astronomer, it was clear that empirical procedures as 'simple' as the determination of the position of a star with some precision were impossible without advanced formal tools, such as a theory of atmospheric refraction. The very task of data gathering could not be fulfilled without using elaborate formal tools that themselves were refined in the process of obtaining data.

To jump to more recent times, cosmology provides another case. While for a long time it was a field with meagre empirical grounding, but with a highly developed mathematical structure, it is now living through a period in which large bodies of new empirical data are being gathered, often of a kind previously unthought-of, such as quasar data or the variations in microwave background. Here the power of mathematical formalism comes into tension with the bulk of new empirical input. For a somewhat contrasting case, one may think of modern molecular genetics, a domain in which the amount of empirical data, produced by scientific machinery, has reached the point that the need for formalization has become essential. Without powerful means of formalization (maybe even mathematical structures), much of the data could easily just get lost within an ocean of others, or become meaningless. So in this instance, it is the empirical overload that is driving the need for a more formal approach.

In all these cases, the 'empirical' and the 'formal' are very closely interlinked, although in different ways. And while there have always been studies of the relation between theory and empirical data (from the induction problem to the recent discussion of the role of models), there have been hardly any attempts to portray and analyze the panorama of various forms of these constellations of close interaction. This state of research is all the more unsatisfactory since both in history and in present day science there are numerous and important cases of such interlinked dependencies of empirical and formal aspects. Hence a research group, centered in Wuppertal/Germany, took the initiative to address these questions. In June 2007, the authors of this introduction, together with Moritz Epple, Helmut Pulte and Erhard Scholz, jointly organized a workshop that focussed, in an exploratory manner, on a better understanding of the various forms of such dense interactions. Four 
of the papers from the workshop that addressed particular historical episodes are assembled in this special issue. Two scientific domains are in the forefront here: hydrodynamics and geomagnetism. In both cases, the papers address a time span that covers significant changes. For hydrodynamics, Olivier Darrigol discusses the relation with regard to the 18 th century and clearly shows the wide gulf between the theoretical approaches and the empirical data. In order to account for punctual points of contact, he proposes the analytic notion of modularity. When Michael Eckert then presents the development of wind tunnels in the 20th century, we see, by contrast, a very close intertwining of the processes used for obtaining data and those needed for developing mathematical structures that allow data handling, and which can even guide the search for further data. A similar transition from a wide gulf to close intertwining becomes visible in the case of geomagnetism. Art Jonkers analyses the processes of data gathering and theorizing in the 17th and 18th centuries and finds that, in many cases, they were separate. However, he makes us aware that talking of theorizing in general is too coarse: the historical case shows different types of theoretical activities that may well have different relations to the process of data-gathering. Looking at the 19 th century, by contrast, Gregory Good presents a different scenario, with theoretical and empirical activities being most closely intertwined, sometimes even politically organized.

In both fields we see the relation between empirical and mathematical activities significantly changing around the turn of the 19th century, with their mutual dependency drastically increasing. This does not only lead to questions of how these changes can be characterized in detail, but also of what made them develop around roughly the same time-questions that can only be answered by further research. Moreover, it would be interesting to see to what degree a distinction between various types of theorizing, as we see in the geomagnetic case, can also be found in the cases of hydrotechnics and hydrodynamics: for example, we do not yet know much about the practitioners' way of conceptualizing and theorizing their problems.

The four papers that make up this special issue of Centaurus attempt, both individually and as a whole, to illustrate the variety of ways in which the empirical and the formal may become intertwined, as well as showing the richness of the research questions that can result from this kind of non- dichotomic consideration.

\section{Friedrich Steinle and Gregor Schiemann University of Wuppertal, Germany}


The Official Journal of the European Society for the History of Science
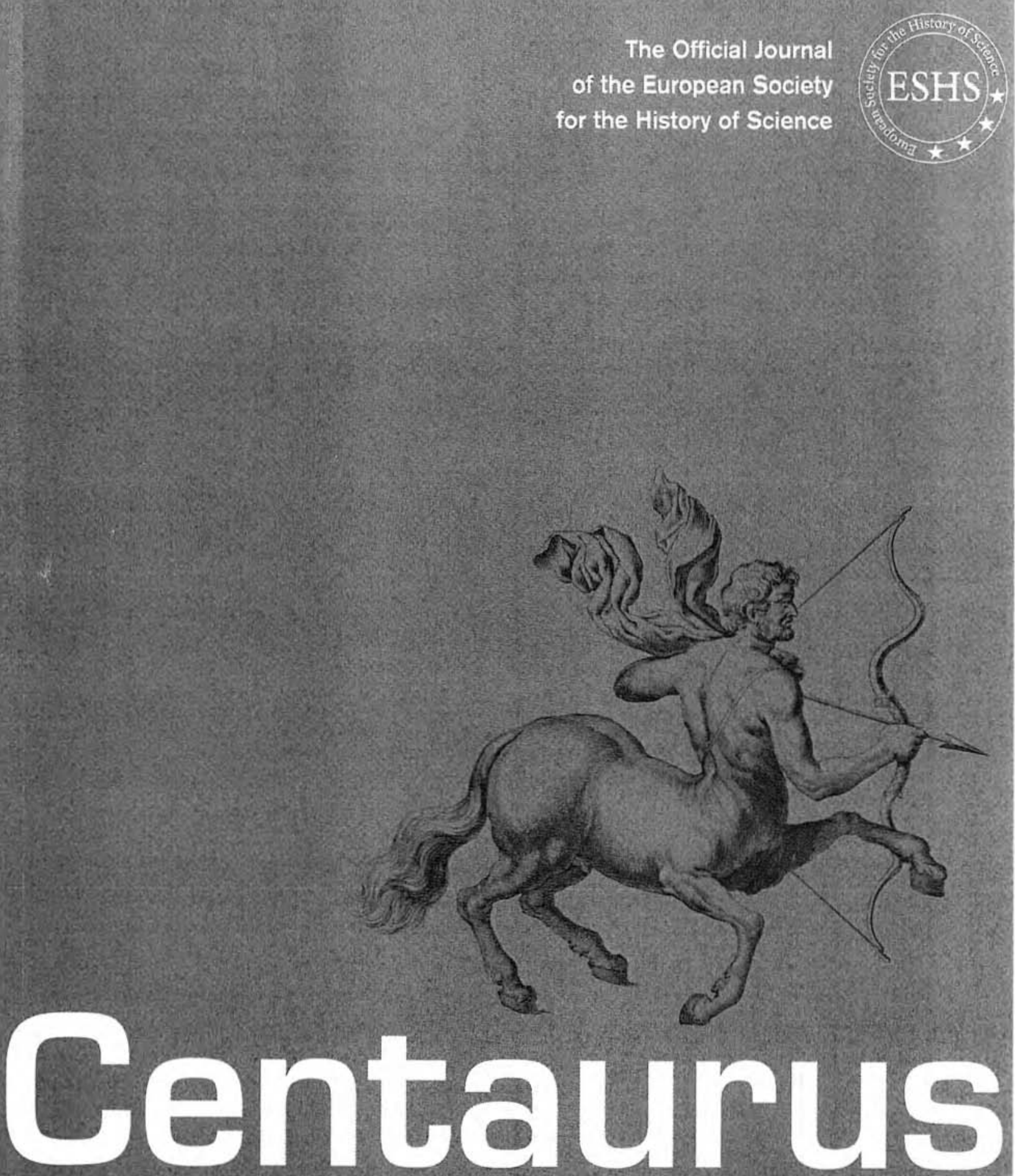

An International Journal of the History of Science and its Cultural Aspects

Volume 50 Number $3 \quad 2008$

\section{(3) WILEY-BLACKWELL}




\section{Centaurus}

Volume 50 Number $3 \quad 2008$ Blackwell Munksgaard

Special Issue: The Empirical and the Formal-Tensions in Scientific Knowledge

\section{CONTENTS}

Steinle F, and Schiemann G.: Introduction: The Empirical and the

Formal-Tensions in Scientific Knowledge ......................211

Darrigol O.: Empirical Challenges and Concept Formation in the

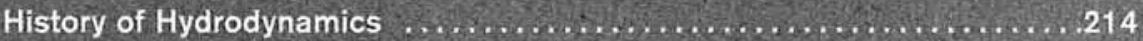

Eckert M: Theory from Wind Tunnels: Empirical Roots of Twentieth Century

Fluid Dynamics ....................................... 233

Jonkers A. R. T.: The Pursuit of Magnetic Shadows: The Formal-Empirical

Dipole Field of Early-Modern Geomagnetism ....................2.254

Good G. A.: Between Data, Mathematical Analysis and Physical Theory:

Research on Earth's Magnetism in the 19 th Century .................290

Social Sciences Citation Index, Arts \& Humanities Citation Index.

Current Contents:

Social \& Behavioral Sciences, Historical Abstracts: 